\title{
Effect of Linear Absorption on Efficiency of SHG in Layered Structure
}

\author{
J. Soltanmohammadi ${ }^{a}$, K. Jamshidi-Ghaleh ${ }^{b, c}$ And H. Masalehdan ${ }^{b}$ \\ ${ }^{a}$ Islamic Azad University, Bonab Branch, Bonab, Iran \\ ${ }^{b}$ Department of Physics, Azarbaijan University of Tarbiat Moallem, Tabriz, Iran \\ ${ }^{c}$ Research Group of Processing and Communication, Azarbaijan Shahid Madani University, Tabriz, Iran
}

\begin{abstract}
The effect of linear absorptions on conversion efficiency of the fundamental radiation to the second harmonic wave in a layered structure is investigated under intensity constant approximation. Behaviour of the conversion efficiency after each layer, up to third layer, is graphically illustrated.
\end{abstract}

DOI: 10.12693/APhysPolA.123.401

PACS: 02.30.-f, 31.15.-p, 31.15.V-

\section{Introduction}

Frequency up-conversion processes have many applications in spectroscopy, optical communications, coherence measurements, etc. On the other hand, periodic layered media such as one-dimensional multilayer photonic crystal find an increasing use in a wide range of telecommunications equipment due to their design flexibility. By adding an additional periodicity to a bulk crystal it is possible to significantly alter its linear and nonlinear optical properties over a narrow wavelength range. In many areas of nonlinear optics the main problem is that of phase matching (PM). On the harmonic conversion process in a bulk medium, under phase mismatching (PMM) condition, because of the spatial biting between different frequencies, the complete conversion of the fundamental wave to the second harmonic is not possible. As the PMM increases, the spatial frequency increases and leads to a decrease on intensity of the generated second harmonic wave [1].

The process of harmonic generation has been investigated in the layered structure [2-8]. In those studies, the linear absorptions of fundamental radiation and second harmonic wave are included in the formalisms. However, at the end when they are presenting their simulation results, the absorption coefficients are set to be zero. Meanwhile, the linear decrease of both, fundamental and generated harmonic waves can be an important parameter in practical results.

In this work, we follow the formalisms that are presented by Tagiev et al. under intensity-constant approximation (ICA) $[7,8]$ in fundamental wave. The results show that the conversion efficiency can be significantly limited by linear absorption at the fundamental radiation and second harmonic wavelength.

\section{Theoretical model and mathematical formulae}

Consider a medium consisting of a structure with $n$ layers and inherently nonlinear property. On each layer, deviation of the phases and linear absorptions of all interacting waves are considered. The subsequent layers can be the same or different linear and nonlinear optical materials. In order to analyse the phenomena, the reduced coupled equations, showing the second harmonic generation (SHG), should be independently considered in each layer. The boundary conditions for each layer are based on the boundary conditions from the previous layer. In other words, the output waves from each layer determine the initial parameters for the next layer.

The standard equations for the coupled complex amplitudes of the fundamental and generated second harmonic waves, inside the $j$-th layer, in the approximation of slow varying amplitudes with considering of the losses are given by

$$
\begin{aligned}
& \frac{\mathrm{d} A_{1 j}}{\mathrm{~d} z}+\alpha_{1 j} A_{1 j}=-\mathrm{i} \gamma_{1 j} A_{2 j} A_{1 j}^{*} \exp \left(-\mathrm{i} \Delta_{j} z\right), \\
& \frac{\mathrm{d} A_{2 j}}{\mathrm{~d} z}+\alpha_{2 j} A_{2 j}=-\mathrm{i} \gamma_{2 j} A_{1 j}^{2} \exp \left(-\mathrm{i} \Delta_{j} z\right),
\end{aligned}
$$

where $A_{1 j}$ and $A_{2 j}$ are complex amplitudes of the fundamental (pump) and second harmonic waves, $\alpha_{1 j}, \alpha_{2 j}$, $\gamma_{1 j}, \gamma_{2 j}$ are the corresponding linear absorption coefficients and nonlinear coupling coefficients of interacting waves at the frequencies of $\omega_{1}=\omega$ and $\omega_{2}=2 \omega$, respectively, in $j$-th layer. $\Delta_{j}=k_{2 j}-2 k_{1 j}$ is the PMM between the interacting waves, $k_{2 j}$ and $k_{1 j}$ are modulus of the corresponding wave vectors. Assuming that in the iterance of the first layer $(z=0)$ there is only the fundamental wave, that is $A_{11}(z=0)=A_{10} \exp \left(\mathrm{i} \varphi_{10}\right)$ and $A_{21}(0)=0$, where $\varphi_{10}$ is the initial phase of the fundamental wave at the input of the medium. Under those initial conditions and applying them to Eq. (1), we obtain $\mathrm{d} A_{21} / \mathrm{d} z=-\mathrm{i} \gamma_{21} A_{11}^{2}(0)$. By using of the initial values and ICA for the fundamental radiation, i.e. $I_{1}(z)=I_{1}(0)=I_{10}=\left|A_{0}\right|^{2}=I_{0}$, the amplitude of the second harmonic wave at the outlet of first layer $\left(z=d_{1}\right)$ with assumption that $\alpha_{21}=2 \alpha_{11}$ is given by

$$
\begin{aligned}
& A_{2}\left(d_{1}\right)=-\mathrm{i} \beta_{21} A_{1}^{2}(0) d_{1} \sin c\left(\Omega_{1} d_{1}\right) \\
& \quad \times \exp \left(\left(2 \varphi_{10}-\alpha_{21}+\frac{\mathrm{i} \Delta_{1}}{2}\right) d_{1}\right),
\end{aligned}
$$

and its corresponding intensity would be as

$$
I_{2}\left(d_{1}\right)=I_{0}^{2} \gamma_{21}^{2} d_{1}^{2} \sin c^{2}\left(\Omega_{1} d_{1}\right) \exp \left(-2 \alpha_{21} d_{1}\right) .
$$

From Eq. (2), the conversion efficiency at the end of first layer is given by 


$$
\eta\left(d_{1}\right)=\frac{I_{2}\left(d_{1}\right)}{I_{0}}=I_{0} \gamma_{21}^{2} d_{1}^{2} \sin c^{2}\left(\Omega_{1} d_{1}\right) \exp \left(-2 \alpha_{21} d_{1}\right) .
$$

Here we have introduced $\Omega_{1}^{2}=2 \Gamma_{1}^{2}+\Delta_{1}^{2} / 4, \Gamma_{1}^{2}=$ $\gamma_{11} \gamma_{21} I_{0}$ and $\sin c x=\sin x / x$.

With the same manner, the intensity of second harmonic wave at the end of the second layer is given by

$$
\begin{aligned}
& I_{2}\left(d_{2}\right)=I_{2}\left(d_{1}\right) \mathrm{e}^{-2 \alpha_{22} d_{2}}\left[\cos ^{2}\left(\Omega_{2} d_{2}\right)\right. \\
& \left.\quad+\left|\frac{\gamma_{22} A_{11}^{2}\left(d_{1}\right) \mathrm{e}^{\mathrm{i}\left(2 \varphi_{11}-\varphi_{21}\right)}}{A_{21}\left(d_{1}\right)}+\frac{\Delta_{2}}{2}\right|^{2} \frac{\sin ^{2}\left(\Omega_{2} d_{2}\right)}{\Omega_{2}^{2}}\right] .
\end{aligned}
$$

Following the same procedures, the conversion efficiency of the fundamental wave to the second harmonic at the outlet of the last layer will be as

$$
\begin{aligned}
& \eta_{2}\left(d_{n}\right)=\eta_{2}\left(d_{n-1}\right)\left[\left(\cos \left(\Omega_{n} d_{n}\right)+c_{a} \gamma_{2 n} \frac{\sin \left(\Omega_{n} d_{n}\right)}{\Omega_{n}}\right)^{2}\right. \\
& +\left(c_{b} \gamma_{2 n} \frac{A_{1}^{2}\left(d_{n-1}\right)}{A_{2}\left(d_{n-1}\right)}+\frac{\Delta_{n}}{2}\right)^{2} \\
& \left.\quad \times \frac{\sin ^{2}\left(\Omega_{n} d_{n}\right)}{\Omega_{n}^{2}}\right] \exp \left(-2 \alpha_{2 n} d_{n}\right) .
\end{aligned}
$$

In Eq. (6), $\Omega_{n}$ is given by $\Omega_{n}^{2}=2 \Gamma_{n}^{2}+\Delta_{n}^{2} / 4$ where $\Gamma_{n}^{2}=\gamma_{2 n} \gamma_{1 n} I_{1}\left(d_{n-1}\right)$ and $c_{a}, c_{b}$ are constants that depend on the linear absorption coefficients $\alpha_{1 n}$ and $\alpha_{2 n}$, nonlinear interaction parameters $\gamma_{1 n}$ and $\gamma_{2 n}$, and the intensities of the interacting waves that their expressions are not shown owing to their bulkiness.

\section{Discussions and results}

Figure 1 shows the conversion efficiency of the fundamental radiation to the second harmonic wave after the first layer for two different length of $d_{1}=1 \mathrm{~cm}$ (a) and $d_{1}=5 \mathrm{~cm}(\mathrm{~b})$. Curves $1-4$ are plotted on various linear absorption coefficients of $0 \mathrm{~cm}^{-1}$ (curve 1), $0.01 \mathrm{~cm}^{-1}$ (curve 2), $0.05 \mathrm{~cm}^{-1}$ (curve 3), and $0.09 \mathrm{~cm}^{-1}$ (curve 4), respectively. It can be seen easily that in the absence

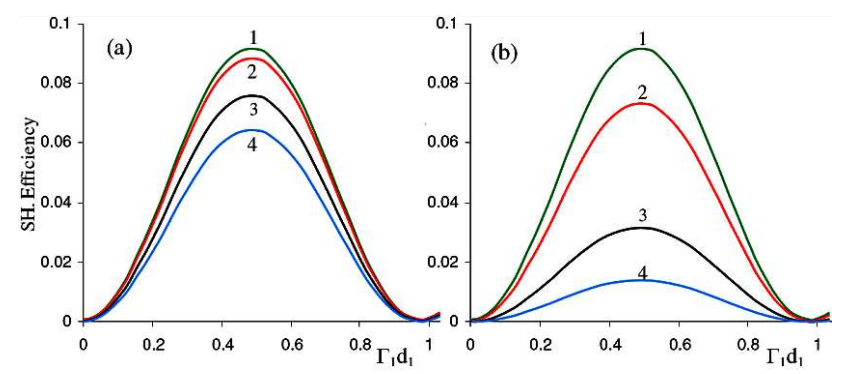

Fig. 1. Conversion efficiency from single layer for two different length of $d_{1}=1 \mathrm{~cm} \mathrm{(a)} \mathrm{and} d_{1}=5 \mathrm{~cm} \mathrm{(b).}$ Curves 1-4 are plotted for linear absorption coefficients of $\alpha_{11}=0 \mathrm{~cm}^{-1}, 0.01 \mathrm{~cm}^{-1}, 0.05 \mathrm{~cm}^{-1}$ and $0.09 \mathrm{~cm}^{-1}$, respectively. Other parameters are used as: $\varphi_{10}=0$, $\alpha_{21}=2 \alpha_{11}, \gamma_{11}=\gamma_{21}$ and $\Delta_{1} / 2 \Gamma_{1}=3$.

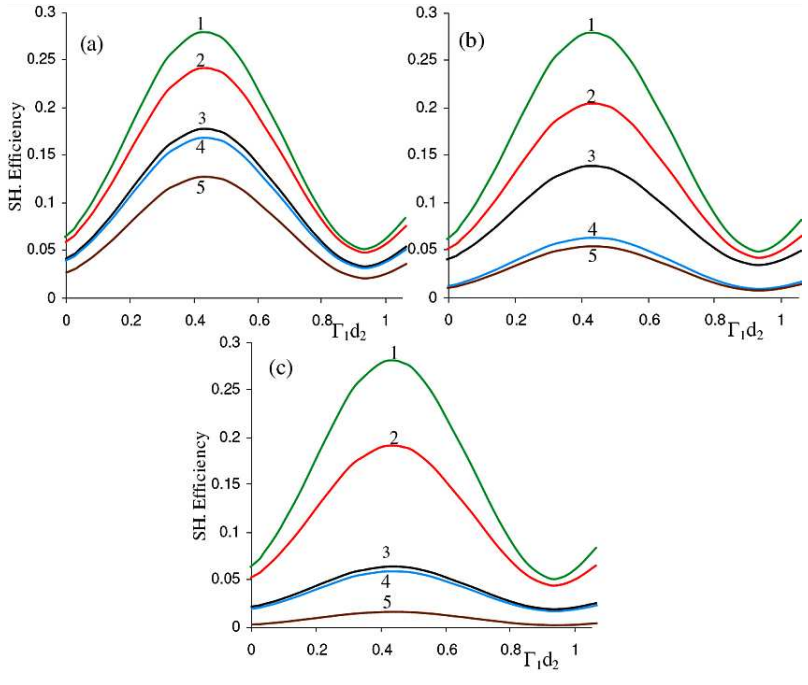

Fig. 2. Conversion efficiency, after the 2nd layer, for thickness of (a) $d_{1}=d_{2}=1 \mathrm{~cm}$, (b) $d_{1}=1 \mathrm{~cm}, d_{2}=$ $5 \mathrm{~cm}$ and $(\mathrm{c}) d_{1}=5 \mathrm{~cm}, d_{2}=1 \mathrm{~cm}$. Curves $1-5$ are plotted for various linear absorption coefficients: $\alpha_{21}=$ $\alpha_{22}=0 \mathrm{~cm}^{-1}, \alpha_{21}=\alpha_{22}=0.01 \mathrm{~cm}^{-1}, \alpha_{21}=0.01 \alpha_{22}=$ $0.09 \mathrm{~cm}^{-1}, \alpha_{21}=0.09 \alpha_{22}=0.01 \mathrm{~cm}^{-1}, 2 \varphi_{11}-\varphi_{21}=2 \pi$ and $\alpha_{21}=\alpha_{22}=0.09 \mathrm{~cm}^{-1}$. The phase mismatching factors are $\Delta_{1} / 2 \Gamma_{1}=-\Delta_{2} / 2 \Gamma_{1}=3$.

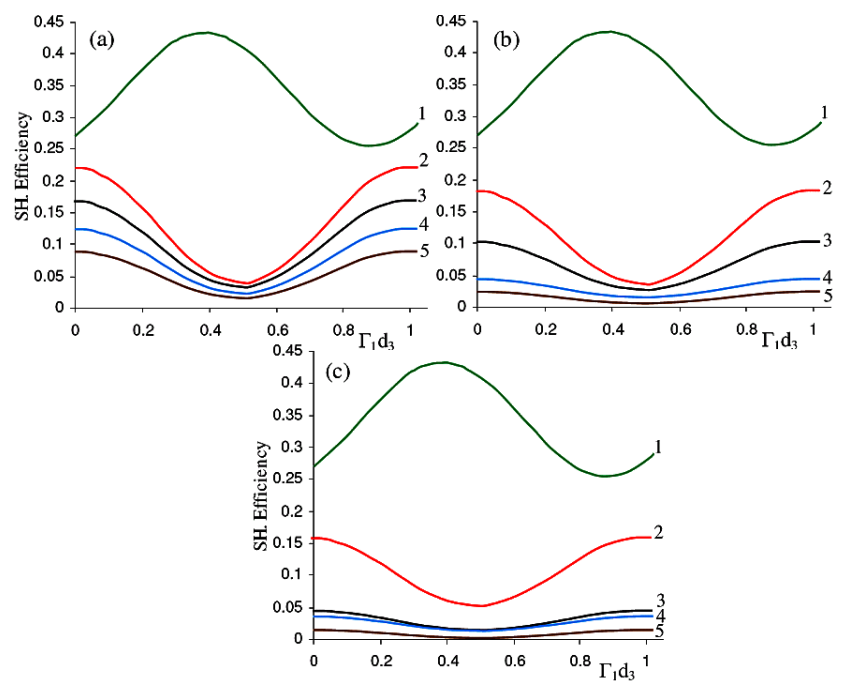

Fig. 3. As Fig. 2, after 3rd layer. The relative phase mismatch factors are set to be $\Delta_{1} / 2 \Gamma=-\Delta_{2} / 2 \Gamma=$ $\Delta_{3} / 2 \Gamma_{1}=3$.

of absorption (curves 1 in Fig. 1a and b) the conversion efficiency is the same for both lengths of $1 \mathrm{~cm}$ and $5 \mathrm{~cm}$. But considering the loss for medium, at thicker sample, with increase of the absorption coefficient, the conversion efficiency is decreased considerably (see curves 4 ). This result was predictable, because thicker sample absorbs more energy of the fundamental wave and the conversion efficiency will be decreased.

The conversion efficiency from two successive layers with thickness of $d_{1}$ and $d_{2}$ is illustrated in Fig. $2 \mathrm{a}-\mathrm{c}$, 
for $d_{1}=1 \mathrm{~cm}, d_{2}=1 \mathrm{~cm} \mathrm{(a),} d_{1}=5 \mathrm{~cm}, d_{2}=1 \mathrm{~cm}(\mathrm{~b})$, $d_{1}=1 \mathrm{~cm}$ and $d_{2}=5 \mathrm{~cm}(\mathrm{c})$. In each figure, curves 1-3 are plotted for linear absorption coefficients of $\alpha_{21}=$ $\alpha_{22}=0 \mathrm{~cm}^{-1}(1), \alpha_{21}=\alpha_{22}=0.01 \mathrm{~cm}^{-1}$ (2) and $\alpha_{21}=\alpha_{22}=0.09 \mathrm{~cm}^{-1}(3)$, respectively. We have used the initial condition of $\alpha_{21}=2 \alpha_{11}, \alpha_{22}=2 \alpha_{12}, \gamma_{11}=\gamma_{21}$ and $\gamma_{12}=\gamma_{22}$.

Other parameters are the same as in Fig. 1. The same method is extended to the $n$-th layer and the conversion efficiency can be obtained with respect to the various parameters of the layers. We have presented the results up to third layer in Figs. 2 and 3.

\section{Summary}

The conversion efficiency of the fundamental radiation to the second harmonic wave in a layered structure was investigated. The effects of linear absorptions on conversion efficiency were graphically illustrated. We assumed that both of the fundamental and generated second harmonic waves are linearly absorbed in each layer. The different linear and nonlinear optical constants for the consisting layers in the structure were considered. The intensity-constant approximation on fundamental wave radiation was applied in the calculations. Behavior of the conversion efficiency after each layer, up to third layer, was graphically illustrated.

\section{References}

[1] Z.H. Tagiev, R.J. Kasumova, Opt. Commun. 268, 311 (2006)

[2] N.S. Kazak, V.K. Pavlenko, Yu.A. Sannikov, J. Appl. Spectrosc. 53, 364 (1990).

[3] Z.H. Tagiev, J. Appl. Spectrosc. 53, 136 (1990)

[4] Z.H. Tagiev, R.J. Kasumova, R.A. Salmanova, N.V. Kerimova, J. Opt. B 3, 84 (2001).

[5] V.G. Dmitriev, L.V. Tarasov, Prikladnaya nelineinaya optika, Radio i Svyaz, Moscow 1982.

[6] R.J. Kasumova, J. Appl. Spectrosc. 78, 659 (2011)

[7] Z.H. Tagiev, R.J. Kasumova, G.A. Safarova, A. Karimi, Appl. Opt. 47, 3681 (2008).

[8] Z.H. Tagiev, R.J. Kasumova, A. Karimi, J. Appl. Spectrosc. 77, 364 (2010). 